\title{
NLRC3 promotes host resistance against Pseudomonas aeruginosa-induced keratitis by promoting the degradation of IRAK1
}

\author{
LITAO GUO $^{1 *}$, QINGZHU KONG ${ }^{2 *}$, ZHIJUN DONG ${ }^{1}$, WEILI DONG ${ }^{1}$, \\ XIAOXIAO FU ${ }^{1}$, LEQI SU ${ }^{3}$ and XIAOBO TAN ${ }^{1}$ \\ Departments of ${ }^{1}$ Ophthalmology and ${ }^{2}$ Orthopedic Trauma, Affiliated Hospital of Chengde Medical University; \\ ${ }^{3}$ Graduate School of Chengde Medical University, Chengde, Hebei 067000, P.R. China
}

Received July 16, 2016; Accepted July 17, 2017

DOI: $10.3892 /$ ijmm.2017.3077

\begin{abstract}
Pseudomonas aeruginosa (PA)-induced keratitis is one of the most common and destructive bacterial diseases. The pathogenesis of PA infections is closely associated with excessive inflammatory responses. Nucleotide oligomerization domain (NOD)-like receptor (NLR) family with caspase activation and recruitment domain (CARD) containing 3 (NLRC3) protein has been implicated as a negative regulator of inflammation and antiviral response, but the role of NLRC3 in PA-induced keratitis has not been described. In the present study, we investigated the effects of NLRC3 in PA-induced keratitis and explored the underlying mechanism. We found that the expression of NLRC3 was decreased in mouse corneas and macrophages after PA infection. Overexpression of NLRC3 significantly attenuated disease progression, inhibited the activation of nuclear factor- $\kappa \mathrm{B}$ signaling and decreased the production of pro-inflammatory cytokines after PA infection. Furthermore, overexpression of NLRC3 promoted K48-linked polyubiquitination and degradation of interleukin-1 receptorassociated kinase 1 (IRAK1). Taken together, we demonstrated that NLRC3 has an anti-inflammatory effect on PA-induced keratitis, which may provide an improved understanding of host resistance to PA infection.
\end{abstract}

\section{Introduction}

Pseudomonas aeruginosa (PA), a Gram-negative opportunistic bacterium, is one of the bacterial species most commonly

Correspondence to: Dr Litao Guo or Dr Xiaobo Tan, Department of Ophthalmology, Affiliated Hospital of Chengde Medical University, Chengde, Hebei 067000, P.R. China

E-mail: guolitao331@163.com

E-mail: tanxiaobo_chengde@163.com

${ }^{*}$ Contributed equally

Key words: NLRC3, Pseudomonas aeruginosa, keratitis, nuclear factor- $\kappa \mathrm{B}$, interleukin-1 receptor-associated kinase 1 isolated from contact lens users with corneal infection such as keratitis. Keratitis caused by PA is characterized by stromal infiltrate, epithelial edema and even tissue destruction (1). Disease progresses rapidly to cause cornea ulceration and can potentially lead to loss of vision from corneal scarring without aggressive treatment (2). Even when viable bacteria are cleared from the cornea, there is often failure to control the immunopathological damage caused by the excessive local inflammation through conventional therapies, such as antibiotic treatment (3). Hence, it is important to develop new therapeutic strategies for the clinical treatment of PA-induced keratitis.

The innate host response to invading microbes such as PA is primarily mediated by innate immune cells, for example macrophages. Once the bacteria break the anatomical barrier, macrophages and other immune cells are quickly recruited to the infection site to engulf invading microorganisms (4). Activated macrophages also produce various pro-inflammatory cytokines such as tumor necrosis factor- $\alpha$ (TNF- $\alpha$ ), interleukin-6 (IL-6) and IL-1 $\beta$, which function to enhance the antibacterial immune response $(5,6)$. Inflammatory mediators promote the elimination of bacteria; nonetheless, if uncontrolled, they may augment the inflammatory response, leading to perforation, tissue damage and loss of vision in the cornea after PA infection. Therefore, it is important to precisely balance pro- and anti-inflammatory responses in ocular immune defense.

The nucleotide oligomerization domain (NOD)-like receptor (NLR) family proteins are intracellular sensors that have many functions, including inflammatory and anti-inflammatory roles (7). Family members share three characteristic domains: the central NOD, N-terminal protein-protein interaction domains and a leucine-rich repeat at the $\mathrm{C}$-terminus $(8,9)$. The most commonly studied subgroup of NLR proteins is composed of the inflammasome-forming NLR proteins (10-12). NACHT, LRR and PYD domains-containing protein 1 (NLRP1), NLRP2, NLRP3, NLR family caspase activation and recruitment domain (CARD) containing 4 (NLRC4), NLRC5, NLRP6 and NLRP7 are reported each able to form an inflammasome complex, which regulates the activation of caspase-1, leading 
to the secretion of biologically active inflammatory cytokines such as IL-1 $\beta$, IL-18 and IL-33, resulting in effects on the process and progression of several inflammatory responses and diseases (11,13-18). Thus, exploring the functions and mechanisms of NLRs has great significance for the remission and treatment of human diseases.

NLRC3, belonging to the NLRC subfamily of NLRs, was firstly identified from human $\mathrm{T}$ lymphocytes and is predominantly expressed in human immune tissues and cells, and it was suggested that NLRC3 functions as a suppressor of $\mathrm{T}$ cell activation (19). In contrast to most NLRs which activate innate immunity, NLRC3 was reported to inhibit LPS-induced toll-like receptor (TLR) signaling by inhibiting the adaptor TNF receptor-associated factor 6 (TRAF6) (20). Recently, NLRC3 was found to be associated with both STING and TBK1 and impeded STING-TBK1 interaction and downstream type I interferon production in response to cytosolic DNA, cyclic di-GMP, and DNA viruses (21). But the function of NLRC3 in PA-induced keratitis and the underlying mechanism still remain unknown.

In the present study, we showed that NLRC3 expression was attenuated in mouse corneas and macrophages in response to PA infection. Overexpression of NLRC3 significantly inhibited the activation of nuclear factor $-\kappa \mathrm{B}(\mathrm{NF}-\kappa \mathrm{B})$ signaling and decreased the production of pro-inflammatory cytokines in PA-infected corneas and macrophages. We also found that NLRC3 could promote K48-ubiquitination and degradation of IL-1 receptor-associated kinase 1 (IRAK1) after PA infection. In conclusion, we revealed an anti-inflammatory role of NLRC3 in PA-induced keratitis in the present study, and suggest NLRC3 as a potential therapeutic target for PA-induced keratitis.

\section{Materials and methods}

Cell culture. Human monocytic leukemia cell line THP-1 and mouse macrophage cell line RAW264.7 were obtained from the American Type Culture Collection (ATCC, Manassas, VA, USA). The cells were cultured at $37^{\circ} \mathrm{C}$ under $5 \% \mathrm{CO}_{2}$ in Dulbecco's modified Eagle's medium (DMEM) supplemented with 10\% FCS (both from Invitrogen Life Technologies, Carlsbad, CA, USA), $100 \mathrm{mg} / \mathrm{ml}$ streptomycin and $100 \mathrm{U} / \mathrm{ml}$ penicillin. Differentiation of THP-1 monocytes to human macrophages was induced by $0.5 \mathrm{mM}$ phorbol 12-myristate 13-acetate (PMA) for $4 \mathrm{~h}$. The differentiated cells were washed 3 times with phosphate-buffered saline (PBS) and followed by the further experiments.

Infection. Eight-week-old female C57BL/6J mice were purchased from Beijing Vital River Laboratory Animal Technology Co., Ltd. (Beijing, China). The protocol of PA infection was performed as previously described $(22,23)$. Mice were anesthetized with ether and placed beneath a stereoscopic microscope at $x 40$ magnification. The cornea of the left eye was wounded with three $1-\mathrm{mm}$ incisions using a sterile 25 -gauge needle. A bacterial suspension (5 $\mu \mathrm{l})$ containing $1 \times 10^{6}$ colonyforming units (CFUs) of PA ATCC strain 19660 was topically applied to the ocular surface. Eyes were examined $24 \mathrm{~h}$ after infection or at other times described here to ensure that mice were similarly infected and to monitor disease.
Clinical examination. Corneal disease was graded as described (24): 0, clear or slight opacity partially or fully covering the pupil; +1 , slight opacity partially or fully covering the anterior segment; +2 , dense opacity partially or fully covering the pupil; +3 , dense opacity covering the entire anterior segment; +4 , corneal perforation or phthisis. The experiments were carried out according to the National Institutes of Health Guide for the Care and Use of Laboratory Animals approved by the Animal Ethics Committee of the Scientific Investigation Board of Chengde Medical University.

Lentivirus preparation and infection. Lentiviral expression plasmids for NLRC3 were constructed by MDL Biotechnology, Co. (Beijing, China). Briefly, the corresponding coding sequence was inserted into the pWPXL vector (Addgene, Cambridge, MA, USA). Lentiviral particles were produced through transfection of pWPXL-NLRC3, pMD2.G and psPAX2 plasmids (all from Addgene) with a proportion of 20:15:7 into 293T cells (ATCC); 3 days later the culture was harvested and enriched by PEG8000. The enriched lentiviral particles (MOI, 50) were used for in vitro cell line infection in the presence of $5 \mu \mathrm{g} / \mathrm{ml}$ of polybrene (Sigma-Aldrich, St. Louis, MO, USA).

Subconjunctival injection of the lentivirus. The protocol was performed as previously described (25). A lentivirus expressing NLRC3 (referred as NLRC3-lentivirus below) or the appropriate control (referred as control-lentivirus below) was subconjunctivally injected into the left eye of C57BL/6J mice $\left(5 \mu \mathrm{l} /\right.$ mouse at a viral titer of $\left.1 \times 10^{8}\right)$ once a week for three times before ocular infection.

Western blot analysis. For western blot analysis, the in vitro cultured cells or corneas were harvested after PA infection and washed three times by ice-cold PBS (MDL Biotechnology, Co.) on ice, and the cells or corneas were lysed with RIPA buffer (MDL Biotechnology, Co.) with proteasome inhibitor cocktail (Roche Diagnostics, Indianapolis, IN, USA), followed by sonication and homogenization. After centrifugation for $15 \mathrm{~min}$ at $13,000 \mathrm{x} \mathrm{g}$, the supernatants were collected and the protein concentration of the lysates was measured using Bio-Rad quantification assay (Bio-Rad Laboratories, Inc., Hercules, CA, USA). Proteins (25 $\mu \mathrm{g})$ were separated using $10 \%$ SDS-PAGE and transferred to a PVDF membrane (Millipore, Billerica, MA, USA). The membrane was then blocked with $2.5 \%$ non-fat dry milk for $1 \mathrm{~h}$. The antibodies for NLRC3 (no. ab77817), IRAK1 (no. ab238) or K48-linked ubiquitin (linkage-specific K48, no. ab140601) (all from Abcam, Cambridge, MA, USA) and the antibodies specific for p65 (no. 8242), phospho-p65 (no. 3033), IKK $\beta$ (no. 2678), phospho-IKK $\beta$ (no. 2697) (all from Cell Signaling Technology, Inc., Beverly, MA, USA) and $\beta$-actin (no. sc58673) (Santa Cruz Biotechnology, Inc., Santa Cruz, CA, USA) were added and incubated overnight at $4^{\circ} \mathrm{C}$. After incubation with the corresponding horseradish peroxidase-conjugated secondary antibody (Santa Cruz Biotechnology, Inc.), the target protein was visualized by enhanced chemiluminescence (Thermo Fisher Scientific, Bremen, Germany). 
Ubiquitination assay. To detect ubiquitination of IRAK1, $10 \mathrm{mM}$ N-ethylmaleimide (Sigma-Aldrich) and 1\% SDS were included in the RIPA buffer (MDL Biotechnology, Co.) with proteasome inhibitor cocktail (Roche Diagnostics). The whole corneas were collected at indicated times after PA infection and were lysed in this RIPA buffer followed by sonication and homogenization. The lysates were denatured at $90^{\circ} \mathrm{C}$ for $5 \mathrm{~min}$. The lysates were diluted with RIPA buffer until the concentration of SDS was decreased to $0.1 \%$. After centrifugation for $15 \mathrm{~min}$ at $13,000 \mathrm{xg}$, supernatants were collected and incubated with Protein G Plus-Agarose Immunoprecipitation reagent (Santa Cruz Biotechnology, Inc.) together with $1 \mu \mathrm{g}$ IRAK1 antibody. After $5 \mathrm{~h}$ of incubation, beads were washed 4 times with immunoprecipitation buffer (MDL Biotechnology, Co.) Immunoprecipitates were eluted by boiling with $1 \%(\mathrm{wt} / \mathrm{vol})$ SDS sample buffer, and the samples were analyzed by immunoblotting with the indicated antibodies.

Bacterial plate counts. Corneas from C57BL/6J mice treated with control-lentivirus or NLRC3-lentivirus were collected at 5 days post-infection ( $\mathrm{n}=5 /$ group/time), and the number of viable bacteria was quantitated as previously described (42). Briefly, individual corneas were homogenized in sterile water containing $0.85 \%(\mathrm{w} / \mathrm{v}) \mathrm{NaCl}$ and $0.25 \%$ BSA. Serial 10 -fold dilutions of the samples were plated on Pseudomonas isolation agar (BD Difco; Becton-Dickinson \& Co., Sparks, MD, USA) in triplicate, and then the plates were incubated overnight at $37^{\circ} \mathrm{C}$. Results are reported as $10^{5} \mathrm{CFU}$ per cornea $\pm \mathrm{SD}$.

RNA isolation and quantitative PCR ( $P P C R)$ analysis. The samples were frozen and mechanically dissociated in TRIzol reagent (Invitrogen Life Technologies). Total RNA was extracted with TRIzol reagent according to the manufacturer's instructions. A LightCycler (ABI PRISM 7000; Applied Biosystems, Foster City, CA, USA) and a SYBR RT-PCR kit (Takara Biotechnology Co., Ltd., Dalian, China) were used for quantitative PCR analysis. GAPDH was used as the internal control, and the $2^{-\Delta \Delta C T}$ method was used to evaluate the relative quantities of each amplified product in the samples. For each qPCR analysis, three technical replicates were performed. Primer sequences used in quantitative PCR are shown in Table I.

ELISA assay. For in vivo studies, corneas from control-lentivirus- and NLRC3-lentivirus-treated mice ( $\mathrm{n}=5 /$ group/time) were individually collected at 5 days post-infection and then homogenized in $0.5 \mathrm{ml}$ of PBS with $0.1 \%$ Tween-20. For in vitro studies, the cell supernatants of the control-lentivirus- and NLRC3-lentivirus-treated RAW264.7 cells were collected at $24 \mathrm{~h}$ post PA challenge. Protein levels of IL-1 $\beta$, IL- 6 and TNF- $\alpha$ were measured using IL-1 $\beta$, IL- 6 , TNF- $\alpha$ ELISA kit (R\&D Systems, Inc., Minneapolis, MN, USA) according to the manufacturer's instructions.

Dual-luciferase reporter gene assays. Luciferase reporter construct containing the NF- $\mathrm{kB}$ promoter region was cloned into pGL3-based vectors, then temporarily transfected with $1 \mu \mathrm{g}$ of the promoter reporter plasmid into the control-lentivirus- or NLRC3-lentivirus-treated RAW264.7 cells using jetPEI ${ }^{\circledR}$-Macrophage (Polyplus-Transfection, New York, NY,
Table I. List of primers used in this study.

\begin{tabular}{ll}
\hline Genes & \multicolumn{1}{c}{ Sequence (5'-3') } \\
\hline NLRC3 & F: CTACCCAAGGCATTCAGCCA \\
(Mus musculus) & R: ACACCTCTTGCTTCCTCGTG \\
NLRC3 & F: CCCTGCAGCAATGACTCAAG \\
(Homo sapiens) & R: TGAAGTCGTGTTCCCTCAGC \\
IL-1 $\beta$ & F: ACCTTCCAGGATGAGGACATGA \\
(Mus musculus) & R: AACGTCACACACCAGCAGGTTA \\
IL-6 & F: ACAACCACGGCCTTCCCTAC \\
(Mus musculus) & R: CATTTCCACGATTTCCCAGA \\
TNF- $\alpha$ & F: GCCACCACGCTCTTCTGTCT \\
(Mus musculus) & R: TGAGGGTCTGGGCCATAGAAC \\
GAPDH & F: AATGACCCCTTCATTGAC \\
(Universal primer) & R: TCCACGACGTACTCAGCGC
\end{tabular}

F, forward; R, reverse; IL-1 $\beta$, interleukin- $1 \beta$; TNF- $\alpha$, tumor necrosis factor- $\alpha$.

USA), and $40 \mathrm{ng}$ of the phRL-TK plasmid were co-transfected into the cells to verify transfection efficiency. After $36 \mathrm{~h}$ of transfection, the luciferase activities were measured on a SpectraMax M5 reader (Molecular Devices, Sunnyvale, CA, USA) using the Dual Luciferase Reporter Assay System (Promega, Madison, WI, USA).

Statistical analysis. The differences in clinical score between the control-lentivirus- and NLRC3-lentivirus-treated corneas were tested by the Mann-Whitney U test at the indicated days post-infection. An unpaired, two-tailed Student's t-test was used to determine the significance of the other assays. Data were considered significant at $\mathrm{P}<0.05$.

\section{Results}

NLRC3 expression is attenuated in mouse corneas after PA infection. To investigate the role of NLRC3 in PA-induced keratitis, we first examined the expression of NLRC 3 in a well-established murine model of PA-induced keratitis. As shown in Fig. 1A and B, the protein level of NLRC3 was consistently expressed in the normal mouse corneas and gradually decreased at 1, 3 and 5 days after PA infection. Consistent with the protein level, the mRNA level of NLRC3 was also decreased in mouse corneas infected with PA (Fig. 1C). Taken together, these results indicated that NLRC3 expression was downregulated at both the mRNA and protein levels in mouse corneas after PA infection, and also illustrated that NLRC3 may be involved in regulating PA-induced keratitis.

NLRC3 expression is decreased in in vitro cultured macrophages after PA infection. During PA infection in the cornea, macrophages and other inflammatory cells infiltrate in the corneal stroma and anterior chamber for the clearance of the bacteria (4-6). To explore the relationship between PA-mediated downregulation of NLRC3 and infiltrated 
A

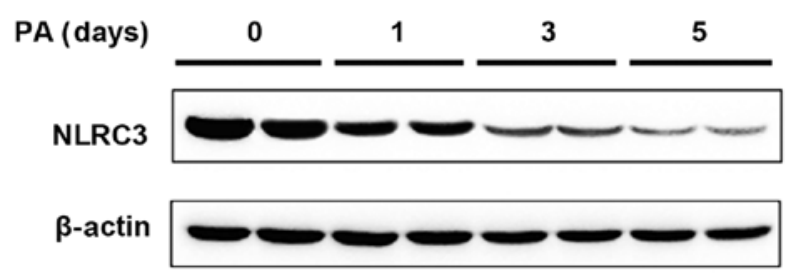

B

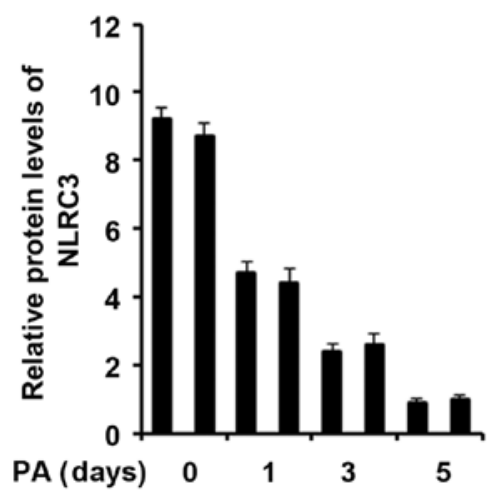

C

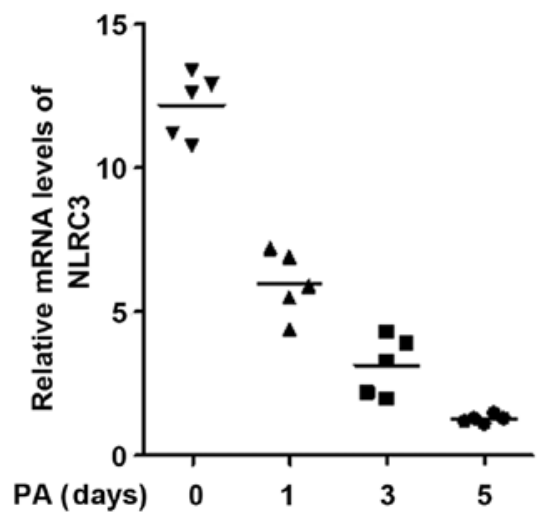

Figure 1. NLRC3 expression is attenuated in mouse corneas after Pseudomonas aeruginosa (PA) infection. (A) Western blot analysis of NLRC3 protein expression in mouse corneas infected with PA for the indicated times, 2 mice/time-point. (B) Band intensity was quantitated and normalized to the $\beta$-actin control in A. (C) qPCR analysis of NLRC3 mRNA expression in mouse corneas infected with PA for the indicated times; 5 mice/time-point. Data are representative of three independent experiments (mean $\pm \mathrm{SD}$ ).

macrophages, we examined the mRNA expression of NLRC3 in macrophage-like RAW264.7 cells and human THP-1-derived macrophages (Fig. 2A). Consistent with the expression data in mouse corneas, mRNA expression of NLRC3 was significantly decreased in RAW264.7 cells and THP-1-derived macrophages infected with PA. Furthermore, we confirmed that the expression level of NLRC3 protein was also significantly downregulated in these in vitro cultured macrophages after PA infection (Fig. 2B and C). Taken together, we hypothesized that the decreased expression of NLRC3 may be related with the infiltration of macrophages.

NLRC3 delays the disease progression of PA-induced keratitis. To determine the potential role of NLRC3 in PA-induced keratitis, C57BL/6 mice were subconjunctivally injected with recombinant lentivirus containing the overexpression plasmid of NLRC3 or a lentivirus containing a control empty vector,
A
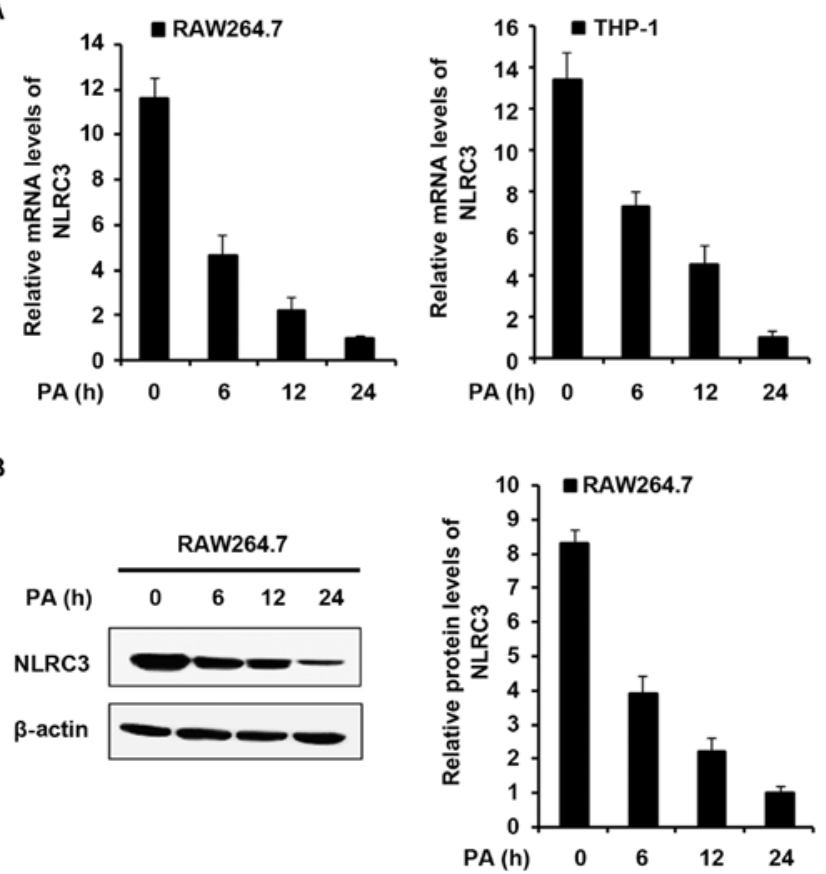

C

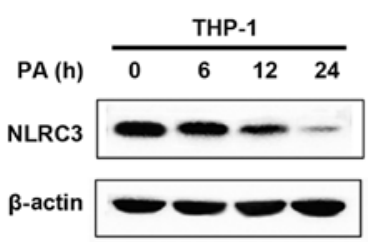

Figure 2. NLRC3 expression is decreased in in vitro cultured macrophages after Pseudomonas aeruginosa (PA) infection. (A) qPCR analysis of NLRC3 mRNA expression in RAW264.7 cells or THP-1-derived macrophages infected with PA for the indicated times. (B) Western blot analysis of NLRC3 protein expression in RAW264.7 cells or (C) THP-1-derived macrophages infected with PA for the indicated times (left); band intensity was quantitated and normalized to the $\beta$-actin control (right). Data are representative of three independent experiments (mean $\pm \mathrm{SD}$ ).

followed by PA infection. The overexpression of NLRC3 in mouse corneas was confirmed by quantitive PCR (qPCR) and western blot analysis (Fig. 3A and B). The overexpression of NLRC3 resulted in less corneal opacity in the PA infected mouse corneas (Fig. 3C). A reduced bacterial load was detected in the infected corneas of NLRC3-overexpressing mice compared to the control group (Fig. 3D). Clinical scores showed that NLRC3-overexpressing mice exhibited less disease severity at 1, 3 and 5 days after PA infection (Fig. 3E). Taken together, these results indicated that overexpression of NLRC3 delayed the disease progression of PA-induced keratitis in mice.

NLRC3 inhibits pro-inflammatory cytokine expression after $P A$ infection. To explore the mechanism by which NLRC3 delays the disease progression of PA-induced keratitis, we first examined the expression of pro-inflammatory cytokines by $\mathrm{qPCR}$ and ELISA in lentivirus-treated corneas and macrophages after PA infection. Overexpression of NLRC3 
A

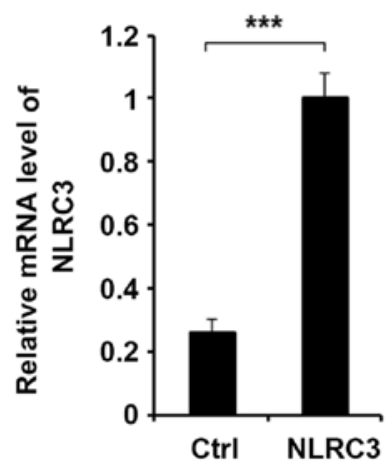

D

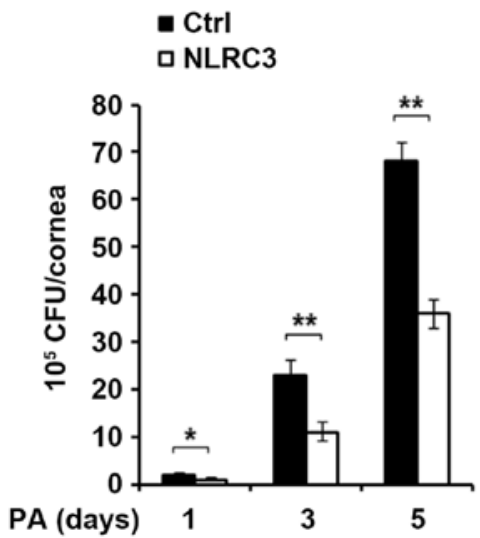

B

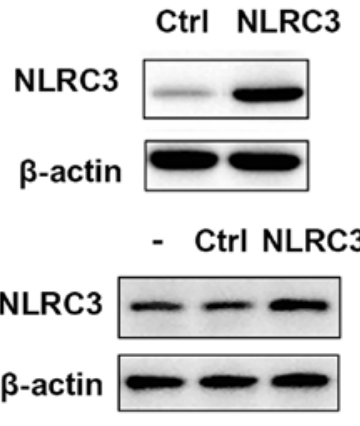

$E$

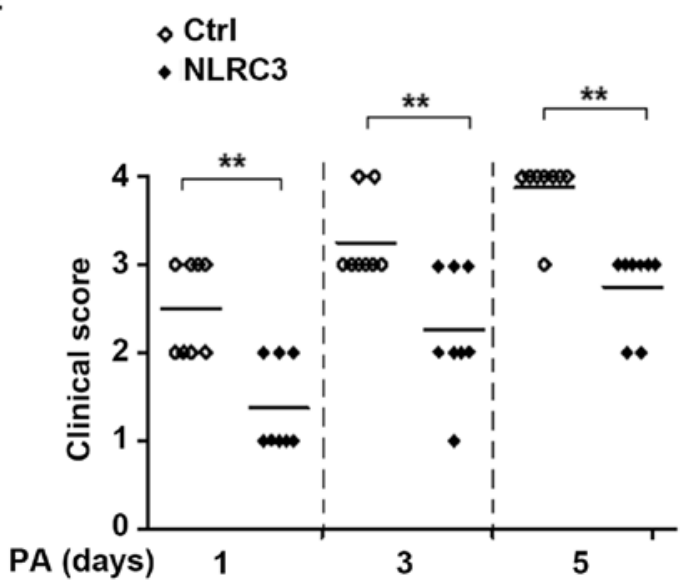

Figure 3. NLRC3 delays the disease progression of Pseudomonas aeruginosa (PA)-induced keratitis. (A) NLRC3 mRNA level and (B, upper image) protein level were examined in the control-lentivirus- or NLRC3-lentivirus-treated mouse corneas at 5 days after PA infection to confirm the efficiency of in vivo overexpression of NLRC3. (B, lower image) The efficiency of in vitro overexpression of NLRC3 in RAW264.7 cells was also confirmed. (C) Images of PA-infected eyes at 5 days post-infection in the control-lentivirus- or NLRC3-lentivirus-treated mice. (D) Bacterial load in the infected corneas was examined by plate count in the control-lentivirus- or NLRC3-lentivirus treated mice at indicated times after PA infection. (E) Clinical scores were recorded in the control-lentivirus- or NLRC3-lentivirus-treated mice at the indicated times after PA infection. Data are representative of three independent experiments (mean \pm SD). ${ }^{*} \mathrm{P}<0.05,{ }^{* *} \mathrm{P}<0.01$ and ${ }^{* * *} \mathrm{P}<0.001$.

significantly inhibited the mRNA level of pro-inflammatory cytokines such as IL-1 $\beta$, IL- 6 and TNF- $\alpha$, in both mouse corneas and in in vitro cultured RAW264.7 macrophages after PA infection (Fig. 4A and B). Consistent with the mRNA data, the ELISA data indicated that protein expression levels of IL-1 $\beta$, IL- 6 and TNF- $\alpha$ were also decreased in the RAW264.7 cells and corneas infected with PA (Fig. 4C and D). Taken together, these results illustrate that NLRC3 inhibited pro-inflammatory cytokine expression after PA infection in vivo and in vitro.

NLRC3 inhibits $N F-\kappa B$ activation after $P A$ infection. $\mathrm{NF}-\kappa \mathrm{B}$ has represented a paradigm for signal transduction and pro-inflammatory cytokine production implicated in numerous diseases including PA-induced keratitis $(26,27)$. Therefore, we ascertained wheather NLRC3 regulates PA-induced keratitis through the NF- $\kappa \mathrm{B}$ signaling pathway. We used DualLuciferase reporter assay to examine the activation of $N F-\kappa B$ signaling, and we found that the promoter activation of $\mathrm{NF}-\kappa \mathrm{B}$ was significantly suppressed in the NLRC3-overexpressing RAW264.7 macrophages compared with the control group after infection with PA for the indicated time (Fig. 5A). In mouse corneas, NLRC3 overexpression also suppressed the phosphorylation of p65 and IKK $\beta$, indicating inhibition of the NF- $\kappa$ B pathway (Fig. 5B and C). Interestingly, no change in NF- $\mathrm{NB}$ activation was detected between the NLRC3 overexpression and control group in the normal uninfected RAW264.7 macrophages or corneas. Taken together, these findings indicated that NLRC3 inhibits $N F-\kappa B$ activation after PA infection.

NLRC 3 promotes degradation and K48-linked polyubiquitination of IRAKI in PA-infected macrophages and mouse corneas. IRAK1 is essential for IL-1, TLR and TNF- $\alpha$-induced $\mathrm{NF}-\kappa \mathrm{B}$ activation (28). After bacterial infection, IRAK1 is phosphorylated and activated by IRAK4, leading to activation of TRAF6-dependent signaling $(29,30)$. NLRC3 was also reported to inhibit LPS-induced TLR signaling via inhibition of the adaptor TRAF6 (20). To further ascertain the mechanism by which NLRC 3 inhibits the activation of NF- $\kappa \mathrm{B}$ signaling, we examined the relationship between NLRC3 and IRAK1. Dual-Luciferase reporter assay was used to examined the activation of $\mathrm{NF}-\kappa \mathrm{B}$ signaling induced by the IRAK1 plasmid. Activation of NF- $\kappa \mathrm{B}$ was significantly inhibited in the NLRC3- 
A

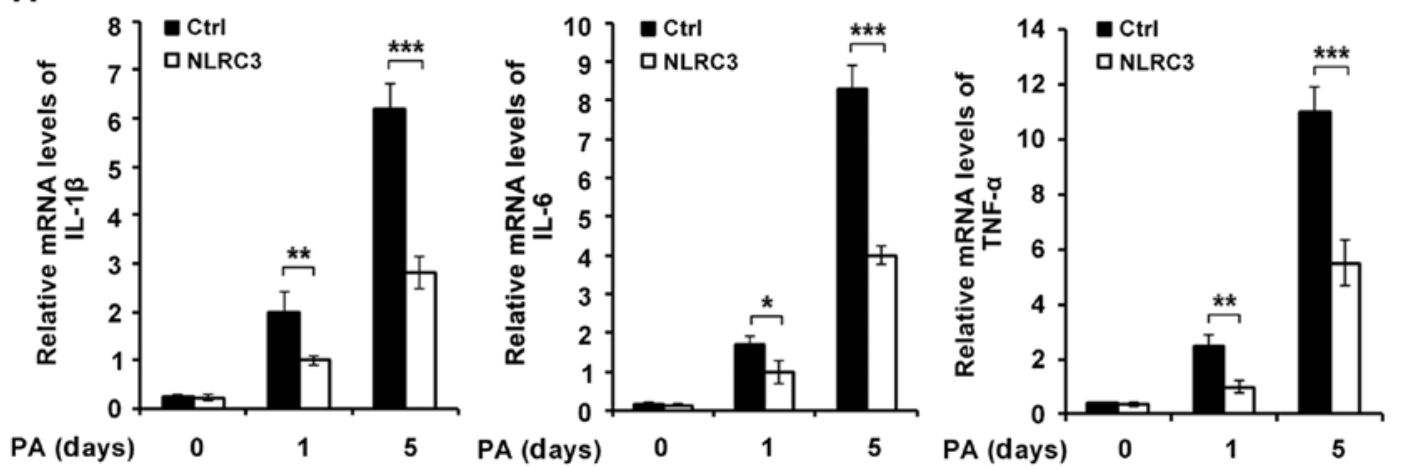

B
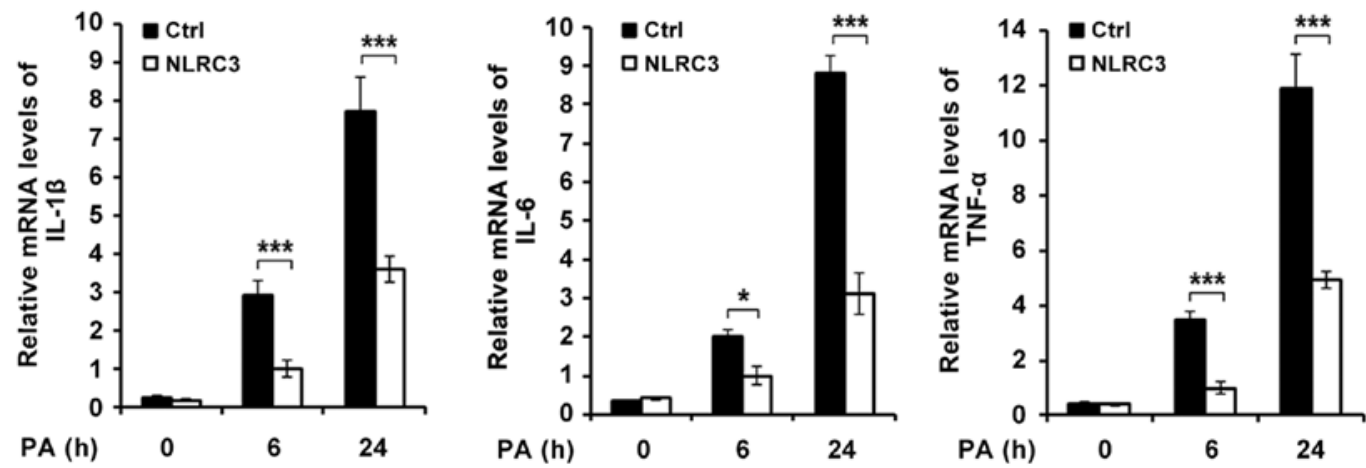

C

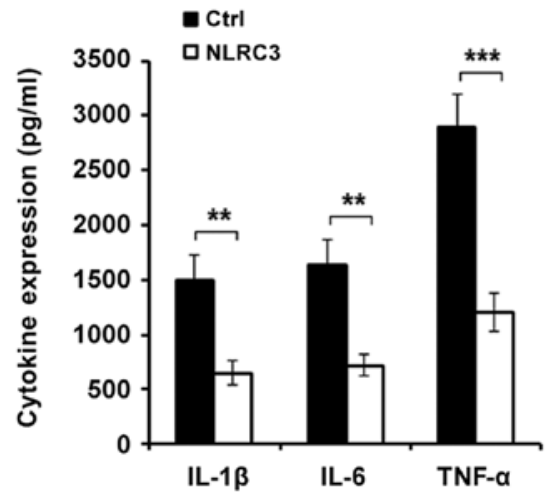

D

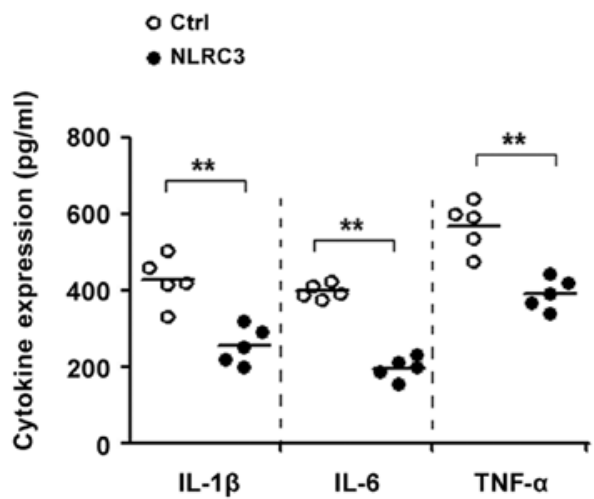

Figure 4. NLRC3 inhibits pro-inflammatory cytokine expression after Pseudomonas aeruginosa (PA) infection. (A) mRNA expression levels of interleukin-1 $\beta$ (IL-1 $\beta$ ), IL-6 and tumor necrosis factor- $\alpha$ (TNF- $\alpha$ ) were examined by qPCR in RAW264.7 cells or (B) THP-1-derived macrophages treated with control-lentivirus or NLRC3-lentivirus followed by PA infection for the indicated times. (C) Protein expression levels of IL-1 $\beta$, IL- 6 and TNF- $\alpha$ determined by ELISA assay in the THP-1-derived macrophages or (D) mouse corneas treated with control-lentivirus or NLRC3-lentivirus at $24 \mathrm{~h}$ (C) or 5 days (D) after $\mathrm{PA}$ infection. Data are representative of three independent experiments (mean $\pm \mathrm{SD}$ ). ${ }^{*} \mathrm{P}<0.05,{ }^{* * *} \mathrm{P}<0.01$ and ${ }^{* * * * *} \mathrm{P}<0.001$.

overexpressing RAW264.7 macrophages compared with the control group after PA infection (Fig. 6A). Overexpression of NLRC3 also significantly decreased the protein level of IRAK1 in the PA-infected RAW264.7 cells (Fig. 6B). K48-linked protein ubiquitination leads to the degradation of the corresponding protein through $26 \mathrm{~S}$ proteasome. Therefore, we examined the K48-linked ubiquitination of IRAK1 in mouse corneas after PA infection. The K48-linked ubiquitination level of IRAK1 was increased in the corneas with overexpressed NLRC3 (Fig. 6C). Taken together, these data indicate that NLRC3 promotes the degradation and K48-linked polyubiquitination of IRAK1 in PA-infected macrophages and mouse corneas.

\section{Discussion}

In the present study, we investigated the expression and function of NLRC3 in mouse corneas and in in vitro cultured macrophages after infection with PA. To the best of our knowledge, this study is the first publication showing the relationship between NLRC3 and PA-induced keratitis, and it also extends our understanding of NLRC3 function.

PA-induced keratitis is a rapidly progressive corneal disease which often leads to inflammatory epithelial edema, corneal ulceration, and even tissue destruction or vision loss (1). It accounts for approximately three quarters of all cases of contact 
A

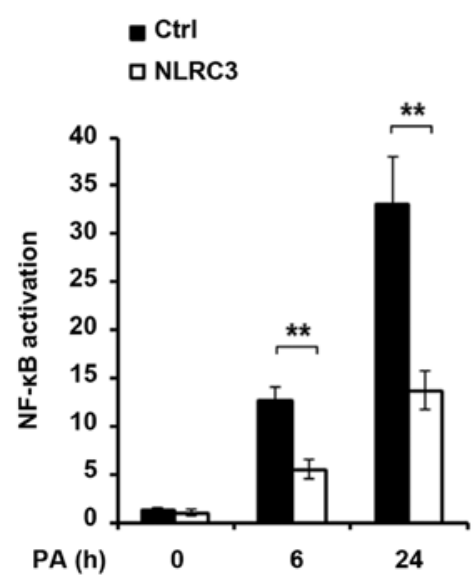

C

- Ctrl

口 NLRC3

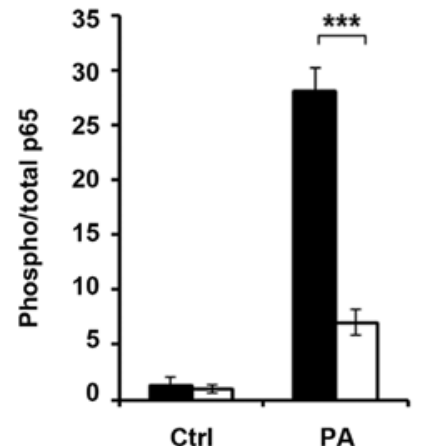

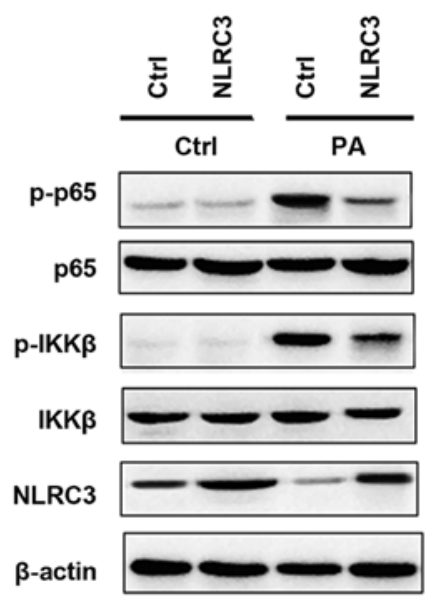

Figure 5. NLRC3 inhibits nuclear factor- $\mathrm{KB}(\mathrm{NF}-\mathrm{\kappa B}$ ) activation after Pseudomonas aeruginosa (PA) infection. (A) RAW264.7 cells were treated with control-lentivirus or NLRC3-lentivirus, followed by transfection with NF- $\mathrm{KB}$ reporter plasmid together with phRL-TK plasmid (internal control); $36 \mathrm{~h}$ later the cells were infected with PA for $24 \mathrm{~h}$ and the activation of NF- $\mathrm{KB}$ promoter was measured by Dual-Luciferase reporter gene assay. (B) Phosphorylation of p65 and IKK $\beta$ was examined by western blot analysis in control-lentivirus- or NLRC3-lentivirus-treated mouse corneas at 5 days after PA infection. (C) Quantification of the protein level of p-p65 and p-IKK $\beta$ in B. Data are representative of three independent experiments (mean \pm SD). ${ }^{* *} \mathrm{P}<0.01$ and ${ }^{* * *} \mathrm{P}<0.001$

A

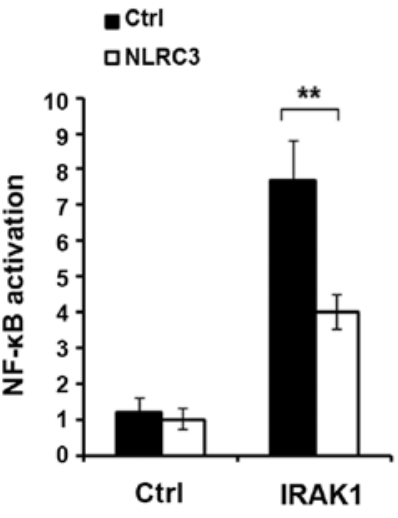

B

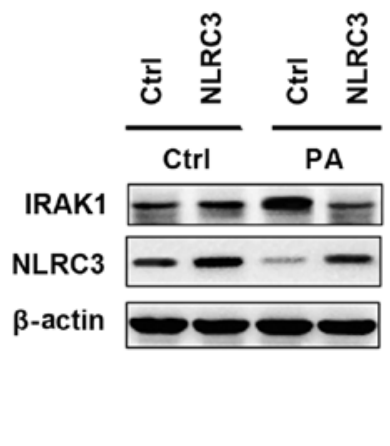

C

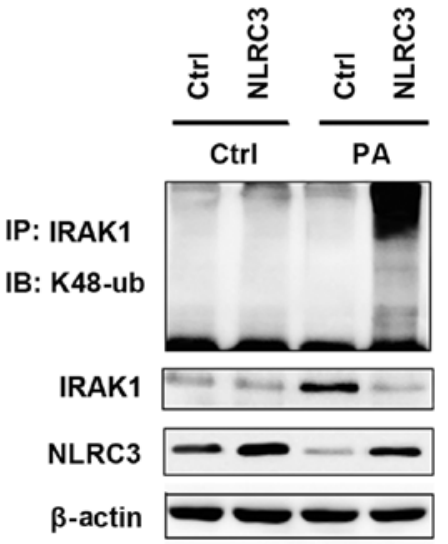

Figure 6. NLRC3 promotes degradation and K48-linked polyubiquitination of interleukin-1 receptor-associated kinase 1 (IRAK1) in Pseudomonas aeruginosa (PA)-infected macrophages and mouse corneas. (A) RAW264.7 cells were treated with control-lentivirus or NLRC3-lentivirus, followed by transfection with nuclear factor- $\mathrm{\kappa B}(\mathrm{NF}-\mathrm{\kappa B})$ reporter plasmid, phRL-TK plasmid, together with control or IRAK1 expression plasmid; $36 \mathrm{~h}$ later the activation of NF- $\mathrm{KB}$ promoter was measured by Dual-Luciferase reporter gene assay. (B) Western blot analysis of IRAK1 expression in RAW264.7 cells treated with control-lentivirus or NLRC3-lentivirus, followed by infection with PA for $24 \mathrm{~h}$. (C) Western blot analysis of K48-linked polyubiquitination level of IRAK1 in control-lentivirus- or NLRC3-lentivirus-treated corneas at 5 days after PA infection. Data are representative of three independent experiments $(\mathrm{mean} \pm \mathrm{SD}) .{ }^{* * *} \mathrm{P}<0.01$. 
lens-associated microbial infection (31). Clinically, doctors often use different types of antibiotics to eradicate the bacteria (32-34). However, even when the viable bacteria are cleared from the cornea, sometimes these treatments do not prevent ocular pathology which is due to the immunopathological damage triggered by the host inflammatory response (35). Therefore, it is of great significance to explore the pathogenesis of keratitis and search for appropriate drug targets.

NLRs are intracellular sensors which comprise a large receptor family of more than 20 members, and nearly half of the NLRs have not been characterized in any detail. Most NLRs positively influence inflammatory responses, particularly the inflammasome NLRs. Recently, physiological roles for inhibitory NLR proteins have been described. Intriguingly, all inhibitory NLRs studied to date have been found to inhibit NF- $\mathrm{B}$ activation. NLRP12 appears to interact with NF- $\kappa \mathrm{B}$-inducing kinase (NIK), TRAF3 and IRAK1. The interactions appear to regulate the phosphorylation of IRAK1 and the degradation of NIK, resulting in inhibition of the NF- $\kappa$ B pathway $(36,37)$. NLRX1 was reported to interact with TRAF6 and diminish its function, leading to the inhibition of NF- $\kappa \mathrm{B}$ activation in transformed cells $(38,39)$. Examination of NLRC3-knockout macrophages showed that there was enhanced $\mathrm{NF}-\kappa \mathrm{B}$ activation downstream of TLR signaling (20). In previous studies, it was also reported that silencing of the NLRP3 inflammasome reduced the level of IL-1 $\beta /$ IL-18 secretion and decreased the level of autophagy, which impaired the elimination of PA in macrophages (40), and NLRC4 (also known as IPAF) inflammasome was shown to play critical roles in the activation of caspase- 1 and IL- $1 \beta$ secretion in PA-infected macrophages (16). However, the roles of NLRs in PA-induced keratitis remain unknown. In the present study, we found that NLRC3 inhibited NF- $\mathrm{B}$ activation in PA-infected macrophages and mouse corneas, and overexpression of NLRC3 significantly inhibited the production of pro-inflammatory cytokines, therefore ameliorating the disease progression of PA-induced keratitis.

Upon ligand binding to TLR or IL-1R, MyD88 interacts with the receptor, and IRAK1 binds to MyD88. Then IRAK4 phosphorylates and activates IRAK1. Phosphorylated IRAK1 disengages from MyD88 and forms a cytosolic IRAK1-TRAF6 complex. TRAF6 then interacts with TAK1, resulting in eventual activation of the NF- $\kappa \mathrm{B}$ and MAPK pathways (41). Thus, the regulation of IRAK1 plays an essential role in $N F-\kappa B$ signaling activation. In this study, we illustrated that NLRC3 could promote degradation of IRAK1. Furthermore, we found that overexpression of NLRC3 significantly increased the K48-linked ubiquitination of IRAK1, which may lead to IRAK1 degradation. These results suggest that NLRC3 is an inhibitory regulator of IRAK1 and enhance our understanding of the regulation of IRAK1 and the NF- $\mathrm{B}$ p pathway.

Interestingly, we found that NLRC3 expression was significantly decreased in PA-infected corneas and macrophages. This phenomenon may be related to the protective role of NLRC3. We supposed that after PA infection, PA could produce different types of virulence factors to inhibit the inflammatory responses of the host, and NLRC3 expression may inhibit these factors therefore leading to the immune escape of PA. Furthermore, we observed that K48-linked ubiquitination was improved by NLRC3 overexpression. We know that NLRC3 only does not have the ability to modulate protein ubiquitination due to the lack of E3 ligase activity. Thus, we hypothesized that there are two possibilities: one is that NLRC3 recruits another E3 ligase which directly promotes the K48-linked ubiquitination of IRAK1; another one is that NLRC3 inhibits the interaction between IRAK1 and its specific deubiquitinase. However, the mechanisms by which NLRC3 regulates IRAK1 ubiquitination still need further study.

In conclusion, in the present study, we found that the expression of NLRC3 was decreased in mouse corneas and macrophages after PA infection. Overexpression of NLRC3 by a lentivirus significantly attenuated PA-induced keratitis progression, inhibited the activation of the $\mathrm{NF}-\kappa \mathrm{B}$ signaling pathway and decreased the production of pro-inflammatory cytokines after PA infection. Furthermore, we observed that overexpression of NLRC3 promoted K48-linked polyubiquitination and degradation of IRAK1 which plays a critical role in NF- $\kappa \mathrm{B}$ activation. We revealed an anti-inflammatory role of NLRC3 in PA-induced keratitis, and suggest NLRC3 as a potential therapeutic target for PA-induced keratitis.

\section{Acknowledgements}

This study was supported by grants from the Natural Science Foundation of Hebei Province (no. H2015406054).

\section{References}

1. Hazlett LD: Corneal response to Pseudomonas aeruginosa infection. Prog Retin Eye Res 23: 1-30, 2004.

2. Wilhelmus KR: Review of clinical experience with microbial keratitis associated with contact lenses. CLAO J 13: 211-214, 1987.

3. Engel LS, Callegan MC, Hobden JA, Reidy JJ, Hill JM and O'Callaghan RJ: Effectiveness of specific antibiotic/steroid combinations for therapy of experimental Pseudomonas aeruginosa keratitis. Curr Eye Res 14: 229-234, 1995.

4. Hazlett LD: Pathogenic mechanisms of P. aeruginosa keratitis: a review of the role of T cells, Langerhans cells, PMN, and cytokines. DNA Cell Biol 21: 383-390, 2002.

5. Kernacki KA, Goebel DJ, Poosch MS and Hazlett LD: Early cytokine and chemokine gene expression during Pseudomonas aeruginosa corneal infection in mice. Infect Immun 66: 376-379, 1998.

6. Xue ML, Thakur A and Willcox M: Gene expression of pro-inflammatory cytokines and chemokines in mouse eye infected with Pseudomonas aeruginosa. Clin Experiment Ophthalmol 30: 196-199, 2002.

7. Eisenbarth SC and Flavell RA: Innate instruction of adaptive immunity revisited: the inflammasome. EMBO Mol Med 1: 92-98, 2009.

8. Shaw PJ, Lamkanfi $M$ and Kanneganti TD: NOD-like receptor (NLR) signaling beyond the inflammasome. Eur $\mathbf{J}$ Immunol 40: 624-627, 2010.

9. Chang SK, Gu Z and Brenner MB: Fibroblast-like synoviocytes in inflammatory arthritis pathology: the emerging role of cadherin-11. Immunol Rev 233: 256-266, 2010.

10. Martinon F, Gaide O, Pétrilli V, Mayor A and Tschopp J: NALP inflammasomes: a central role in innate immunity. Semin Immunopathol 29: 213-229, 2007.

11. Davis BK, Roberts RA, Huang MT, Willingham SB, Conti BJ, Brickey WJ, Barker BR, Kwan M, Taxman DJ, Accavitti-Loper MA, et al: Cutting edge: NLRC5-dependent activation of the inflammasome. J Immunol 186: 1333-1337, 2011.

12. Lamkanfi $M$ and Dixit VM: Modulation of inflammasome pathways by bacterial and viral pathogens. J Immunol 187: 597-602, 2011.

13. Masters SL, Gerlic M, Metcalf D, Preston S, Pellegrini M, O'Donnell JA, McArthur K, Baldwin TM, Chevrier S, Nowell CJ, et al: NLRP1 inflammasome activation induces pyroptosis of hematopoietic progenitor cells. Immunity 37: 1009-1023, 2012. 
14. Minkiewicz J, de Rivero Vaccari JP and Keane RW: Human astrocytes express a novel NLRP2 inflammasome. Glia 61: $1113-1121,2013$.

15. Duewell P, Kono H, Rayner KJ, Sirois CM, Vladimer G, Bauernfeind FG, Abela GS, Franchi L, Nuñez G, Schnurr M, et al: NLRP3 inflammasomes are required for atherogenesis and activated by cholesterol crystals. Nature 464: $1357-1361,2010$.

16. Sutterwala FS, Mijares LA, Li L, Ogura Y, Kazmierczak BI and Flavell RA: Immune recognition of Pseudomonas aeruginosa mediated by the IPAF/NLRC4 inflammasome. J Exp Med 204: 3235-3245, 2007.

17. Elinav E, Strowig T, Kau AL, Henao-Mejia J, Thaiss CA, Booth CJ, Peaper DR, Bertin J, Eisenbarth SC, Gordon JI, et al NLRP6 inflammasome regulates colonic microbial ecology and risk for colitis. Cell 145: 745-757, 2011.

18. Khare S, Dorfleutner A, Bryan NB, Yun C, Radian AD, de Almeida L, Rojanasakul Y and Stehlik C: An NLRP7-containing inflammasome mediates recognition of microbial lipopeptides in human macrophages. Immunity 36 : 464-476, 2012.

19. Conti BJ, Davis BK, Zhang J, O'connor W Jr, Williams KL and Ting JP: CATERPILLER 16.2 (CLR16.2), a novel NBD/LRR family member that negatively regulates $\mathrm{T}$ cell function. $\mathrm{J}$ Biol Chem 280: 18375-18385, 2005 .

20. Schneider M, Zimmermann AG, Roberts RA, Zhang L, Swanson KV, Wen H, Davis BK, Allen IC, Holl EK, Ye Z, et al: The innate immune sensor NLRC3 attenuates toll-like receptor signaling via modification of the signaling adaptor TRAF6 and transcription factor NF- $\kappa$ B. Nat Immunol 13: 823-831, 2012.

21. Zhang L, Mo J, Swanson KV, Wen H, Petrucelli A, Gregory SM, Zhang Z, Schneider M, Jiang Y, Fitzgerald KA, et al: NLRC3, a member of the NLR family of proteins, is a negative regulator of innate immune signaling induced by the DNA sensor STING. Immunity 40: 329-341, 2014

22. Yang K, Wu M, Li M, Li D, Peng A, Nie X, Sun M, Wang J, Wu Y, Deng Q, et al: miR-155 suppresses bacterial clearance in Pseudomonas aeruginosa-induced keratitis by targeting Rheb. J Infect Dis 210: 89-98, 2014.

23. Huang X, Barrett RP, McClellan SA and Hazlett LD: Silencing toll-like receptor-9 in Pseudomonas aeruginosa keratitis. Invest Ophthalmol Vis Sci 46: 4209-4216, 2005.

24. Hazlett LD, McClellan S, Kwon B and Barrett R: Increased severity of Pseudomonas aeruginosa corneal infection in strains of mice designated as Th1 versus Th2 responsive. Invest Ophthalmol Vis Sci 41: 805-810, 2000.

25. Chen K, Yin L, Nie X, Deng Q, Wu Y, Zhu M, Li D, Li M, Wu $\mathrm{M}$ and Huang $\mathrm{X}$ : $\beta$-Catenin promotes host resistance against Pseudomonas aeruginosa keratitis. J Infect 67: 584-594, 2013.

26. Karin M and Lin A: NF-kappaB at the crossroads of life and death. Nat Immunol 3: 221-227, 2002.

27. Sun Y, Karmakar M, Roy S, Ramadan RT, Williams SR Howell S, Shive CL, Han Y, Stopford CM, Rietsch A, et al: TLR4 and TLR5 on corneal macrophages regulate Pseudomonas aeruginosa keratitis by signaling through MyD88-dependent and -independent pathways. J Immunol 185: 4272-4283, 2010.
28. Luftig M,Prinarakis E, Yasui T, Tsichritzis T, Cahir-McFarland E, Inoue J, Nakano H, Mak TW, Yeh WC, Li X, et al: Epstein-Barr virus latent membrane protein 1 activation of NF-kappaB through IRAK1 and TRAF6. Proc Natl Acad Sci USA 100: $15595-15600,2003$.

29. Suzuki N, Suzuki S, Duncan GS, Millar DG, Wada T, Mirtsos C, Takada H, Wakeham A, Itie A, Li S, et al: Severe impairment of interleukin-1 and toll-like receptor signalling in mice lacking IRAK-4. Nature 416: 750-756, 2002.

30. Cao Z, Xiong J, Takeuchi M, Kurama T and Goeddel DV: TRAF6 is a signal transducer for interleukin-1. Nature 383: 443-446, 1996.

31. Liesegang TJ: Contact lens-related microbial keratitis: part I: epidemiology. Cornea 16: 125-131, 1997.

32. Kowalski RP, Romanowski EG, Mah FS, Shanks RM and Gordon YJ: Topical levofloxacin $1.5 \%$ overcomes in vitro resistance in rabbit keratitis models. Acta Ophthalmol 88: e120-e125, 2010

33. McCormick C, Caballero A, Tang A, Balzli C, Song J and O'Callaghan R: Effectiveness of a new tobramycin $(0.3 \%)$ and dexamethasone $(0.05 \%)$ formulation in the treatment of experimental Pseudomonas keratitis. Curr Med Res Opin 24: $1569-1575,2008$

34. Mohammadpour M, Mohajernezhadfard Z, Khodabande A and Vahedi P: Antibiotic susceptibility patterns of Pseudomonas corneal ulcers in contact lens wearers. Middle East Afr J Ophthalmol 18: 228-231, 2011.

35. Cowell BA, Willcox MD, Hobden JA, Schneider RP, Tout S and Hazlett LD: An ocular strain of Pseudomonas aeruginosa is inflammatory but not virulent in the scarified mouse model. Exp Eye Res 67: 347-356, 1998.

36. Allen IC, Wilson JE, Schneider M, Lich JD, Roberts RA, Arthur JC, Woodford RM, Davis BK, Uronis JM, Herfarth HH, et al: NLRP12 suppresses colon inflammation and tumorigenesis through the negative regulation of noncanonical NF- $\kappa B$ signaling. Immunity 36: 742-754, 2012.

37. Ye Z, Lich JD, Moore CB, Duncan JA, Williams KL and Ting JP. ATP binding by monarch-1/NLRP12 is critical for its inhibitory function. Mol Cell Biol 28: 1841-1850, 2008.

38. Allen IC, Moore CB, Schneider M, Lei Y, Davis BK, Scull MA, Gris D, Roney KE, Zimmermann AG, Bowzard JB, et al: NLRX1 protein attenuates inflammatory responses to infection by interfering with the RIG-I-MAVS and TRAF6-NF- $\mathrm{BB}$ signaling pathways. Immunity 34: 854-865, 2011.

39. Xia X, Cui J, Wang HY, Zhu L, Matsueda S, Wang Q, Yang X, Hong J, Songyang Z, Chen ZJ, et al: NLRX1 negatively regulates TLR-induced NF- $\mathrm{B}$ B signaling by targeting TRAF6 and IKK. Immunity 34: 843-853, 2011.

40. Deng Q, Wang Y, Zhang Y, Li M, Li D, Huang X, Wu Y, Pu J and Wu M: Pseudomonas aeruginosa triggers macrophage autophagy to escape intracellular killing by activation of the NLRP3 inflammasome. Infect Immun 84: 56-66, 2015.

41. Gottipati S, Rao NL and Fung-Leung WP: IRAK1: a critical signaling mediator of innate immunity. Cell Signal 20: 269-276, 2008 